\title{
On the contribution of point sources to the Galactic ridge X-ray emission
}

\author{
M. Revnivtsev ${ }^{1,2}$ and S. Sazonov ${ }^{1,2}$ \\ 1 Max-Planck-Institute für Astrophysik, Karl-Schwarzschild-Str. 1, 85740 Garching bei München, Germany \\ e-mail: mikej@mpa-garching.mpg.de \\ 2 Space Research Institute, Russian Academy of Sciences, Profsoyuznaya 84/32, 117997 Moscow, Russia
}

Received 23 February 2007 / Accepted 18 June 2007

\begin{abstract}
We analyzed deep Chandra observations of the Galactic plane region centered at $l=28.55^{\circ}, b=-0.03^{\circ}$ with the aim to obtain the best possible constraints on the contribution of weak point sources to the Galactic ridge X-ray emission (GRXE) in this region. We demonstrate that the vast majority of the detected sources are Galactic in origin and are probably cataclysmic variables and coronally active stars. We use the number-flux function of detected sources to constrain the luminosity function of Galactic X-ray sources in the range $10^{30}-10^{32} \mathrm{erg} \mathrm{s}^{-1}$ and find good agreement with the luminosity function of sources in the Solar vicinity. The fraction of the total flux at energies $1-7 \mathrm{keV}$ resolved into point sources at the current sensitivity level is $\sim 25 \%$. Excluding the expected contribution of extragalactic sources, $\sim 19 \%$ of the GRXE is due to point Galactic sources with interstellar absorption corrected fluxes higher than $1.2 \times 10^{-15} \mathrm{erg} \mathrm{s}^{-1} \mathrm{~cm}^{-2}$ in the energy band $1-7 \mathrm{keV}$.
\end{abstract}

Key words. stars: binaries: general - Galaxy: disk - X-rays: general - X-rays: stars

\section{Introduction}

The origin of the Galactic ridge X-ray emission (GRXE) has been a long-standing problem of X-ray astronomy. Since the discovery of this emission in the late 1970s (Cooke et al. 1970; Bleach et al. 1972; Worrall et al. 1982), different explanations have been proposed for its nature. These can be divided into two major branches: i) the GRXE is truly diffuse, i.e. it is generated in the interstellar medium (see e.g. Koyama et al. 1986, 1989; Tanaka 2002; Ebisawa et al. 2005), or ii) it is the integrated emission of faint Galactic point sources (Worrall \& Marshall 1983; Koyama et al. 1986; Ottmann \& Schmitt 1992; Mukai \& Shiokawa 1993; Revnivtsev et al. 2006b).

The main problem with the former hypothesis is the difficulty of keeping plasma that is apparently thermal and hot (>5-10 keV) (Koyama et al. 1986, 1989; Tanaka 2002; Koyama et al. 2006) within a thin layer along the Galactic plane (see e.g. Worrall et al. 1982; Warwick et al. 1985; Yamauchi \& Koyama 1993; Revnivtsev et al. 2006b).

The latter scenario recently received strong support from work of our team (Revnivtsev et al. 2006b; Sazonov et al. 2006; Krivonos et al. 2006; Revnivtsev et al. 2006a). In particular, we obtained high-quality maps of the GRXE that showed striking similarity with the near-infrared map representing the distribution of stellar mass over the Milky Way. We concluded that throughout the Galaxy the GRXE volume emissivity is proportional to the stellar mass density. Furthemore, we showed that the GRXE emissivity per unit stellar mass is consistent with the cumulative emissivity of known classes of weak X-ray sources in the Solar vicinity - cataclysmic variables and coronally active stars (Sazonov et al. 2006).

We recently used deep Chandra observations of a field in the close vicinity of the Galactic Center (GC) to demonstrate that at least $40-50 \%$ of the total $\mathrm{X}$-ray emission from that region is created by point sources (Revnivtsev et al. 2006c). It is known that the properties of the X-ray emission from the GC region are very similar to those of the large-scale GRXE, suggesting that both have the same physical origin (e.g. Tanaka 2002). However, as the space density of stars near the GC is 3-4 orders of magnitude higher than is typical for the Galactic plane (GP), one could expect that truly diffuse X-ray emission, should such exist, might provide a more significant contribution to the GRXE from the GP.

This motivated us to study one of the deepest Chandra observations of the GP, centered at $l=28.55^{\circ}, b=-0.03^{\circ}$, with the aim to estimate the contribution of resolved Galactic point sources to the total X-ray flux from this direction. This observation was previously analyzed by Ebisawa et al. (2005).

\section{Data reduction}

We used the Chandra observation (OBSID 2298) of the GP region in the direction $l=28.5548^{\circ}, b=-0.026^{\circ}$, with a total exposure time of $\sim 102 \mathrm{ks}$. In order to maximize the sensitivity to point sources, we restricted our analysis to the data collected within $4^{\prime}$ radius around the aim point of the telescope, where its angular resolution is better than $1^{\prime \prime}(F W H M)$. At larger distances from the aimpoint the size of the point spread function of Chadnra significantly increases ( 2.6 times at $4^{\prime}$ distance from the aimpoint) and the sensitivity drops by more then 1.5 times (e.g. Muno et al. 2006), therefore we do not consider these regions below. The solid angle of the field of our study is thus $\sim 4.25 \times 10^{-6}$ ster. Note that we did not analyze existing Chandra observations of a nearby GP region (OBSIDs 949 and 1523), because the central 4 '-area of this field does not overlap with the region of our study, making it impossible to increase the point source sensitivity, which is key to us, by adding these extra data. 
The data were reduced following a standard procedure fully described in Vikhlinin et al. (2005). The only difference is that the detector background was now modeled using the stowed dataset (http://cxc.harvard.edu/ contrib/maxim/stowed). Point sources were detected using the wavelet decomposition package wvdecomp of ZHTOOLS software (Vikhlinin et al. 1998) ${ }^{1}$.

Due to the significant drop in the Chandra sensitivity at energies $E>2 \mathrm{keV}$ and especially at $E>7 \mathrm{keV}$, and because of the strong effect of interstellar photoabsorption in the GP at $E<1 \mathrm{keV}$, we used the energy band 1-7 keV for our analysis. A total of $6.5 \mathrm{kcnts}$ in this energy band were accumulated from the studied area of $0.01396 \mathrm{sq}$. deg over $102 \mathrm{ks}$. This implies the total observed X-ray flux $F_{1-7} \mathrm{keV}$, observed $=$ $(1.0 \pm 0.1) \times 10^{-12} \mathrm{erg} \mathrm{s}^{-1} \mathrm{~cm}^{-2}$. For conversion of the count rate into the flux we used the simplest best fit model to the observed data points - a power law in energy band $1-7 \mathrm{keV}$ with the photon index $\Gamma=0.7$.

After constructing the number-flux function of deteced sources, we corrected it for the incompleteness near the detection threshold using the procedure developed for our GC study (Revnivtsev et al. 2006c). In essence, Poisson fluctuations in the number of counts detected from a weak source effectively reduce the probability of its detection in a given observation (see e.g. Hasinger et al. 1993; Kenter \& Murray 2003). To correct for this "leakage" of sources under the detection threshold, we derived the allowed range of intrinsic $\log N-\log S$ functions of sources by simulating mock Chandra images for a large number of such functions and checking which of these images are consistent with the measured number-flux relation. The multifold of allowed intrinsic $\log N-\log S$ functions was then used to determine the resolved fraction of the GRXE.

The studied Chandra field is characterized by heavy interstellar absorption. The total column density of atomic and molecular gas through the Galactic disk in this direction is estimated at $\sim 6 \times 10^{22} \mathrm{H}$ atoms per $\mathrm{cm}^{2}$ (Ebisawa et al. 2005). The Galactic sources in the field are expected to be located at widely differing distances, from our close vicinity all the way to the outer boundary of the Milky Way, whereas the distribution of the absorbing gas within the sampled cone of the Galaxy is poorly known. Ebisawa et al. (2005) made an attempt to estimate typical column densities from stacked Chandra spectra of subsets of sources detected in this field and found values ranging from $\sim 0.5 \times 10^{22}$ to $>5 \times 10^{22} \mathrm{~cm}^{-2}$. In view of the remaining uncertainty, we assume in our analysis that all Galactic X-ray sources in the field are characterized by the same absorbing column density $N_{\mathrm{H}}=2 \times 10^{22} \mathrm{~cm}^{-2}$, while all extragalactic ones by $N_{\mathrm{H}}=6 \times 10^{22} \mathrm{~cm}^{-2}$.

To make a direct comparison of the source counts in the GP region with those in typical extragalactic fields (maximally devoid of Galactic sources), we also analyzed Chandra observations of the Deep Fields South (CDFS, OBSID 5021) and North (CDFN, OBSID 3389) in exactly the same manner as the GP data. To have the same exposure time as for the GP field, we used only $\sim 100 \mathrm{ks}$ of the available (ultra-deep) observations for each of these extragalactic fields. However, even in this case the number of detected extragalactic sources in the GP region is expected to be significatly reduced compared to the CDFS and CDFN by the strong interstellar absorption.

\footnotetext{
1 http://hea-www.harvard.edu/saord/zhtools/
}

\section{Expected number-flux functions of Galactic and extragalactic sources}

The majority of weak X-ray sources $\left(L_{\mathrm{x}}<10^{34} \mathrm{erg} \mathrm{s}^{-1}\right)$ in our Galaxy are cataclysmic variables and coronally active binary stars (e.g. Vaiana 1981; Sazonov et al. 2006). These classes of sources are expected to be distributed over the Milky Way just like ordinary stars (with the notable exception of globular clusters where the relative fraction of X-ray sources may be different due to the greatly increased role of dynamical processes in forming such systems). Such proportionality between the space density of weak X-ray sources and that of stars has indeed been observed (see e.g. Muno et al. 2006; Revnivtsev et al. 2006c). This allows us to predict the distribution of X-ray sources along our studied light of sight and consequently the expected $\log N-\log S$ function for a given source luminosity function, e.g. for that measured in the Solar vicinity (Sazonov et al. 2006).

In estimating the contribution of extragalactic sources to the number-flux function measured in the GP field, we preferred to carry out a direct comparison with $\sim 100$ ks subsets of CDFN and CDFS observations, rather than relying on published $\log N-\log S$ functions of extaragalactic sources (e.g. Moretti et al. 2002). This allows us to use the same energy band (1-7 keV) for detecting sources, thus avoiding any rescaling and its associated uncertainties. Also, number-flux relations constructed from these observations should be affected by the leakage of weak sources (see above) similarly to the GP observation, faciliating comparison of the measured $\log N-\log S$ curves.

\subsection{Galactic disk model}

In order to make a prediction for the number-flux function of Galactic X-ray sources in the studied GP region, we should take into account the spatial distribution of stars (which have been shown to be good tracers of weak X-ray sources) along the line of sight. For this purpose we should adopt some mass model of the Galaxy. Since our observation is directed relatively far from the GC $\left(l \approx 28.5^{\circ}\right)$, we do not expect any significant contribution from sources residing in the Galactic bulge. Thus, it is sufficient to consider only the disk component of the Galaxy.

There is general agreement that the space density of stars in the Galactic disk is exponentially declining (with scaleheight $R_{\text {disk }}$ ) with Galactocentric radius $R$ and that there is a similar decline of density with radius within some minimal radius $R_{\mathrm{m}}$ due to the presence of the Galactic bulge/bar inside this radius (e.g. Freudenreich 1998). We therefore adopt below the following mass model of the Galactic disk:

$\rho_{\text {disk }}(R)=\rho_{0, \text { disk }} \exp \left[-\left(\frac{R_{\mathrm{m}}}{R}\right)^{3}-\frac{R}{R_{\text {disk }}}\right]$,

where $\rho_{0, \text { disk }}=5.5 M_{\odot} \mathrm{pc}^{-3}, R_{\mathrm{m}}=2.5 \mathrm{kpc}$, and $R_{\text {disk }}=2.2 \mathrm{kpc}$. We additionally assumed that the stellar space density abruptly drops outside a Galactocentric radius of $10 \mathrm{kpc}$ and that the distance between the Sun and the GC is $8.0 \mathrm{kpc}$. As our observation is aimed almost exactly at the Galactic plane and the angular size of the studied region is tiny $\left(\sim 4^{\prime}\right)$, it is appropriate to ignore the vertical distribution of stars in the Milky Way.

Parameter of the adopted model are slightly different from those used in Sazonov et al. (2006) and Revnivtsev et al. (2006b) due to special requirement to predict correct near-infrared (NIR) surface brightness value at the region of our study $\left(l \sim 28.5^{\circ}\right)$. Good correspondence of the NIR surface brightness profile to that observed by COBE/DIRBE (corrected for the interstellar extinction following Revnivtsev et al. 2006a) is seen in Fig. 1. 


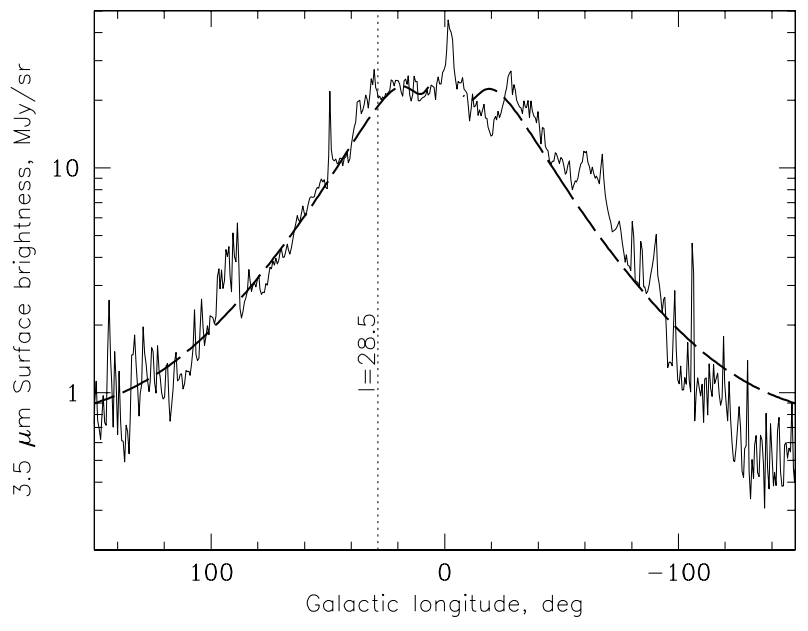

Fig. 1. Profile of the near-infrared brightness of the Galaxy along its plane as seen by $C O B E / D I R B E$ at $3.5 \mu \mathrm{m}$. The dashed line is a profile predicted by the Galaxy mass model adopted in this paper.

\subsection{Cumulative characteristics at $I=28.5^{\circ}$}

The extinction corrected surface brightness of the Galactic disk at $3.5 \mu \mathrm{m}$ in the direction of our observation $\left(l \sim 28.5^{\circ}, b \sim\right.$ $0.03^{\circ}$ ) is $I_{3.5 \mu \mathrm{m}} \sim 21 \pm 4 \mathrm{MJy} / \mathrm{sr}$ (a significant fraction of the quoted uncertainty is associated with the correction of the $C O B E / D I R B E$ data for interstellar extinction), or $^{2}(1.4 \pm 0.3) \times$ $10^{-6} \mathrm{erg} \mathrm{s}^{-1} \mathrm{~cm}^{-2} \mathrm{deg}^{-2}$. Given the area $\left(1.39 \times 10^{-2} \mathrm{deg}^{-2}\right)$ of the studied Chandra field, the total NIR flux from this region $F_{3.5 \mu \mathrm{m}}=(2.0 \pm 0.4) \times 10^{-8} \mathrm{erg} \mathrm{s}^{-1} \mathrm{~cm}^{-2}$.

From our study of the large-scale distribution of the GRXE with RXTE we found the ratio of the NIR surface brightness to the GRXE intensity in the $3-20 \mathrm{keV}$ band to be $(4.1 \pm 0.3) \times 10^{-5}$ (Revnivtsev et al. 2006b). Assuming that the GRXE spectrum is a power-law with photon index $\Gamma=2$, we can rescale this factor to the $1-7 \mathrm{keV}$ band: $F_{1-7 \mathrm{keV}} / F_{3.5 \mu \mathrm{m}}=(4.2 \pm 0.3) \times 10^{-5}$. Multiplying this ratio by the measured NIR flux yields the expected GRXE intensity in the considered direction: $(5.9 \pm 1.2) \times$ $10^{-11} \mathrm{erg} \mathrm{s}^{-1} \mathrm{~cm}^{-2} \mathrm{deg}^{-2}$. Consequently, the GRXE flux from the entire Chandra field is expected to be $F_{1-7 \mathrm{keV}}=(8.4 \pm$ 1.8) $\times 10^{-13} \mathrm{erg} \mathrm{s}^{-1} \mathrm{~cm}^{-2}$. This estimate does not take into account the significant interstellar absorption in the studied direction. Considering column densities $N_{\mathrm{H}} \sim 0.5-4 \times 10^{22} \mathrm{~cm}^{-2}$ to be typical of Galactic X-ray sources in this field (see the discussion of this issue in Sect. 2 above), the expected absorbed GRXE flux $F_{1-7} \mathrm{keV}$, absorbed $\sim(4-7) \times 10^{-13} \mathrm{erg} \mathrm{s}^{-1} \mathrm{~cm}^{-2}$.

The above estimate still does not take into account the contribution of extragalactic sources. The all-sky average intensity of the cosmic X-ray background (CXB) is $I_{2-10 \mathrm{keV}} \sim$ $2 \times 10^{-11} \mathrm{erg} \mathrm{s}^{-1} \mathrm{~cm}^{-2} \mathrm{deg}^{-2}$ (e.g. Revnivtsev et al. 2003, 2005; Hickox \& Markevitch 2006), or $I_{1-7} \mathrm{keV} \sim 1.8 \times$ $10^{-11} \mathrm{erg} \mathrm{s}^{-1} \mathrm{~cm}^{-2} \mathrm{deg}^{-2}$ (assuming a power-law spectrum with $\Gamma=1.4$ ). This translates into a flux $F_{1-7 \mathrm{keV}} \sim 2.5 \times$ $10^{-13} \mathrm{erg} \mathrm{s}^{-1} \mathrm{~cm}^{-2}$ for our Chandra field. Taking into account that the full Galactic column density in the direction of our study is $N_{\mathrm{H}} \sim 6 \times 10^{22} \mathrm{~cm}^{-2}$, the CXB contribution to the observed $\mathrm{X}$-ray flux is expected to be $\sim 1.2 \times 10^{-13} \mathrm{erg} \mathrm{s}^{-1} \mathrm{~cm}^{-2}$. Therefore, the total (GRXE plus CXB) X-ray flux from the studied region should be $F_{1-7} \mathrm{keV}$, expected $\sim(5-8) \times 10^{-13} \mathrm{erg} \mathrm{s}^{-1} \mathrm{~cm}^{-2}$. This is compatible with the flux actually measured by

\footnotetext{
2 We adopted the DIRBE bandwidths from http://lambda.gsfc.nasa.gov/product/cobe/ about_dirbe.cfm.
}

Chandra: $F_{1-7 \mathrm{keV} \text {, observed }}=(1.0 \pm 0.1) \times 10^{-12} \mathrm{erg} \mathrm{s}^{-1} \mathrm{~cm}^{-2}$. We should stress here that correcting for the interstellar absorption is very important for the $1-7 \mathrm{keV}$ energy band but is not straightforward and subject to significant uncertainties due to possible variations in the absorption along the line of sight and across the studied field.

The consistency of cumulative NIR and X-ray characteristics of the studied Chandra field provides additional support to our adopted Galaxy model, which is used in the subsequent analysis. We emphasize that it is crucial that the adopted mass model correctly reproduces the NIR flux from the considered direction of the GP.

\subsection{Luminosity function of weak Galactic X-ray sources}

Despite the long history of studying weak $\left(L_{\mathrm{x}}<10^{34-35} \mathrm{erg} \mathrm{s}^{-1}\right)$ Galactic X-ray sources, their luminosity function remained poorly constrained until lately. We recently used two all-sky X-ray surveys (the RXTE slew survey in the 3-20 keV band and the ROSAT all-sky survey below $2 \mathrm{keV}$ ) to construct a luminosity function of X-ray sources located near the Sun in the broad range $10^{27}-10^{34} \mathrm{erg} \mathrm{s}^{-1}$ (Sazonov et al. 2006). Below we use this luminosity function in conjunction with the Galaxy model described above for predicting the number-flux function of sources in the studied GP region.

\section{Results}

\subsection{X-ray images}

If there were no significant population of weak Galactic X-ray sources, one could expect the surface density of point sources to be similar in different regions of the sky observed by Chandra to the same depth ${ }^{3}$. However, if we compare the Chandra image of the GP region with those of the CDFN and CDFS obtained with the same exposure (Fig. 2), we clearly see that the surface density of point sources is much higher in the GP region.

This difference becomes even more pronounced if we take into account that due to the considerable interstellar absorption in the direction of $l \sim 28.5^{\circ}, b \sim 0.0^{\circ}$, the number of detected extragalactic sources in that field is expected to be significantly reduced compared to the CDFN and CDFS. For extragalactic sources with fluxes $10^{-15}-10^{-14} \mathrm{erg} \mathrm{s}^{-1} \mathrm{~cm}^{-2}$ in the 1-7 keV energy band, whose spectra can typically be described as power laws with photon indexes $\Gamma \sim 1.4-1.8$ (e.g. Hickox \& Markevitch 2006), the Galactic absorption with $N_{\mathrm{H}} \sim$ $6 \times 10^{22} \mathrm{~cm}^{-2}$ is expected to suppress the Chandra count rates in the $1-7 \mathrm{keV}$ band by a large factor of $\sim 4.3-5.3$ (note that since absorption mostly removes photons with energies $\sim 1-2 \mathrm{keV}$, the corresponding decrease in the energy flux at $1-7 \mathrm{keV}$ is much smaller). Therefore, for a given exposure time, Chandra should detect in the CDFN and CDFS roughly 5 times as many extragalactic sources as in the GP field.

The much higher surface density of sources in the GP region is further reflected in the $\log N-\log S$ function of detected sources (see Fig. 5 below). We conclude that in the Galactic plane weak point X-ray sources of Galactic origin greatly outnumber extragalactic ones.

\footnotetext{
${ }^{3}$ Although counts of extragalactic sources exhibit small field-to-field variations due to the large-scale structure of the Universe (see e.g. Voss \& Gilfanov 2006; Cappelluti et al. 2007).
} 

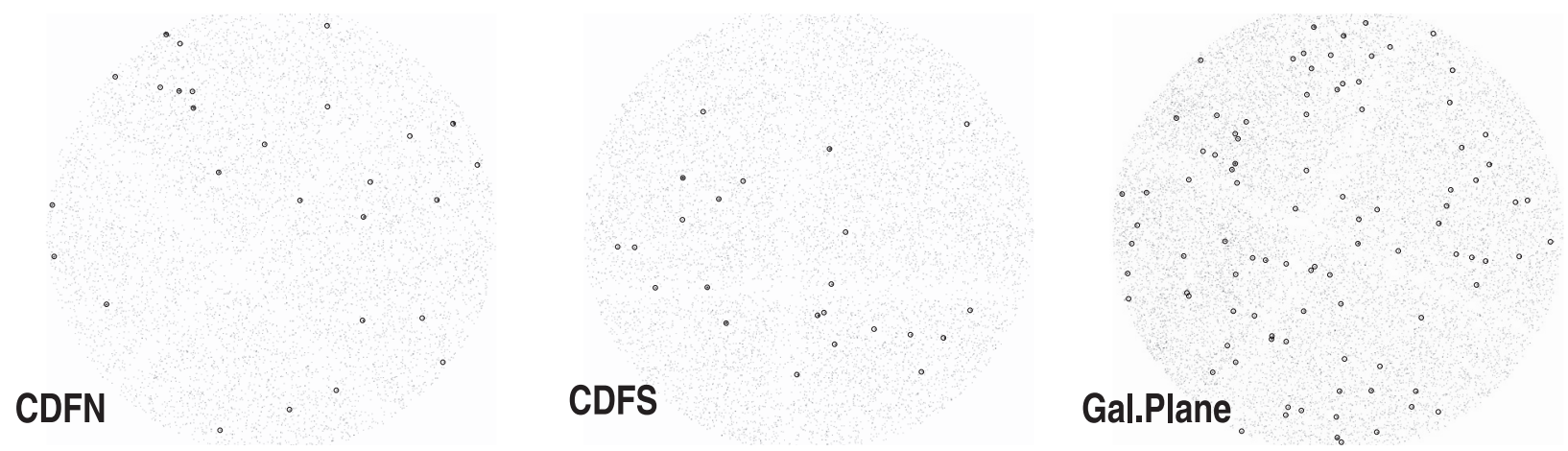

Fig. 2. Comparison of the raw Chandra images (1-7 keV) of three sky regions. From left to right - Chandra Deep Field North (CDFN), Chandra Deep Field South (CDFS), and Galactic plane region at $l \sim 28.5^{\circ}$. For each of the CDFN and CDFS only $\sim 100$ ks of the available observations are used and the same circular region around the telescope's aim point is chosen as for the GP field. Even with this equal setup for all three images, Chandra's sensitivity to extragalactic sources is expected to be much worse in the GP region compared to both extragalactic fields. In reality, the surface density of point sources is much higher in the GP region, clearly indicating the presence of a large number of Galactic sources in the field.

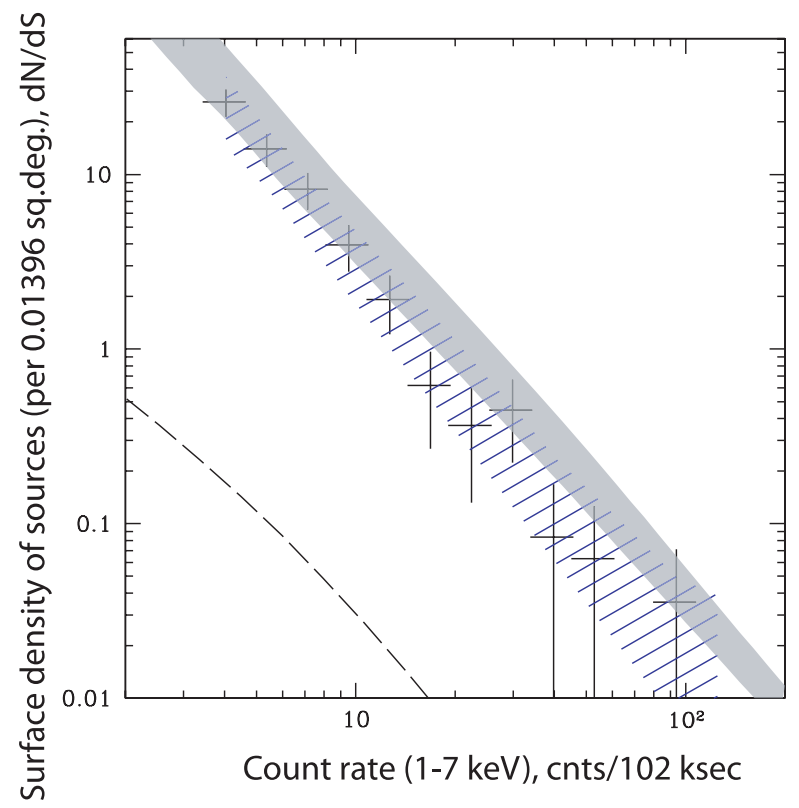

Fig. 3. Differential number-flux function of sources measured in the GP region and corrected for the incompleteness at low count rates (points with error bars). The hatched region represents the manifold of number-flux functions allowed by the data (see main text and the corresponding range of allowed luminosity functions in Fig. 4). The gray band shows the expected range of number-flux functions based on the luminosity function of weak X-ray sources in the Solar vicinity from Sazonov et al. (2006) and including the predicted contribution of extragalactic sources. The width of this region is determined by the $1 \sigma$ uncertainties in the luminosity function of local sources. Count rates of Galactic sources were reduced to take into account the typical interstellar absorption in the GP region $\left(N_{\mathrm{H}} \sim 2 \times 10^{22} \mathrm{~cm}^{-2}\right.$, see main text). The expected $\log N-\log S$ distribution of extragalactic sources (dashed line) was also modified by the Galactic absorption $\left(N_{\mathrm{H}} \sim 6 \times 10^{22} \mathrm{~cm}^{-2}\right)$. One Chandra count of used $102 \mathrm{ks}$ observation corresponds to unabsorbed fluxes of $2.5 \times 10^{-16} \mathrm{erg} \mathrm{s}^{-1} \mathrm{~cm}^{-2}$ and $5 \times 10^{-16} \mathrm{erg} \mathrm{s}^{-1} \mathrm{~cm}^{-2}$ for Galactic $\left(N_{\mathrm{H}} \sim 2 \times 10^{22} \mathrm{~cm}^{-2}, \Gamma=2.0\right)$ and extragalactic $\left(N_{\mathrm{H}} \sim 6 \times 10^{22} \mathrm{~cm}^{-2}\right.$, $\Gamma=1.8)$ sources, respectively.

\section{2. $\log N-\log S$ distribution}

We now address the number-flux function of detected sources. In Fig. 3 we show the differential $\log N-\log S$ distribution measured by Chandra in the GP region and corrected for the incompleteness near the detection threshold (see Sect. 2). These data are compared with the results of our modelling described below.

To make a prediction for the number-flux function, we convolved the luminosity function of weak X-ray sources measured in the Solar vicinity (Sazonov et al. 2006) with the Galactic disk model described in Sect. 3.1. To convert the $2-10 \mathrm{keV}$ luminosity function of Sazonov et al. (2006) into the 1-7 keV band, we assumed a power-law spectrum with $\Gamma=2.0$. In addition, to take into account the interstellar absorption in the considered direction, we multipled all predicted $1-7 \mathrm{keV}$ count rates by a factor of 0.34 , which corresponds to a power-law spectrum with $\Gamma=2.0$ absorbed by neutral gas with column density $N_{\mathrm{H}}=2 \times 10^{22} \mathrm{~cm}^{-2}$. As we discussed in Sect. 2 , this is only a rough (but reasonable) approximation of the true situation, as in reality there is a large scatter in $N_{\mathrm{H}}$ values from source to source.

As regards the expected contribution of extragalactic sources, we estimated it from the $\log N-\log S$ curves measured in the CDFN and CDFS by applying an absorption correction as appropriate for our GP field $\left(N_{\mathrm{H}} \sim 6 \times 10^{22} \mathrm{~cm}^{-2}\right)$. Adding this expected extragalatic contribution (shown by the dashed line in Fig. 3) to the expected contribution of Galactic sources yields the expected range of $\log N-\log S$ functions, shown in gray in Fig. 3. There is apparently a good agreement between the expected and observed distributions. The apparent small difference between them may well be caused by some inaccuracy in our correction for the interstellar absorption.

On the other hand, we can use the observed numberflux function to put constraints on the luminosity function of point sources in the studied GP region. To this end, we assumed that the luminosity function in a $L^{2} \mathrm{~d} N / \mathrm{d} L$ representation can be described by a set of constants in the following intervals: $10^{28}-10^{29}, 10^{29}-10^{30}, 10^{30}-10^{31}, 10^{31}-10^{32}$, and $10^{32}-10^{33} \mathrm{erg} \mathrm{s}^{-1}$. By allowing these constants to vary and convolving the resulting luminosity functions with our Galactic disk model, we obtained a manifold of trial number-flux functions of sources. These were then compared in terms of the reduced $\chi^{2}$ value $\left(\chi^{2}\right.$ per degree of freedom) with the observed $\log N-\log S$ function (taking into account the estimated contribution of extragalactic sources). Those trial luminosity functions that resulted in a reduced $\chi^{2}$ of less than 1.5 were regarded as allowed by the data. The resulting allowed range of numberflux functions (including extragalactic sources) is shown as the dashed area in Fig. 3, and the corresponding range of acceptable luminosity functions of Galactic sources is shown as the dashed 


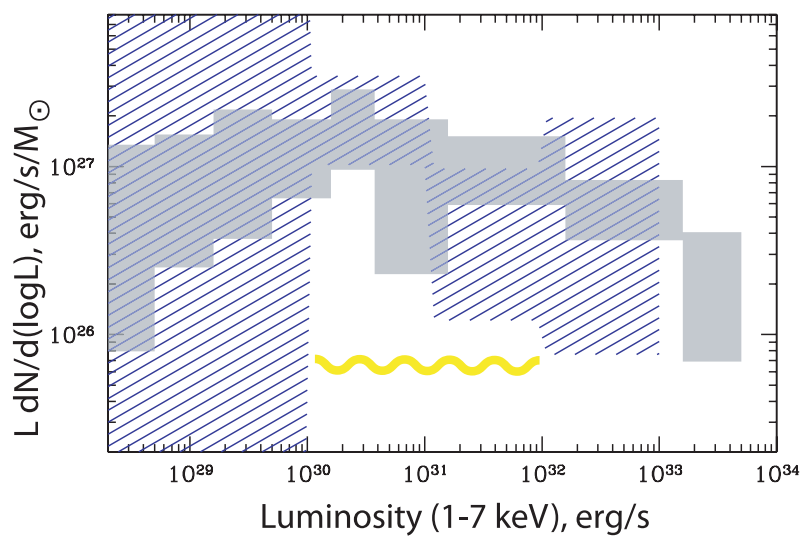

Fig. 4. Range of luminosity functions of Galactic X-ray sources in the GP region (hatched area) allowed by the measured number-flux function (see Fig. 3). This is compared with the luminosity function (gray area) of weak X-ray sources in the Solar neighborhood (Sazonov et al. 2006). For the latter we included the uncertainty in the fraction of young single stars in the studied GP region (relative to cataclysmic variables and coronally active binary stars, see Sazonov et al. 2006 for details). Specifically, we allowed this contribution to vary from zero to the value measured near the Sun. Note that we effectively probe only the luminosity range $10^{30}-10^{32} \mathrm{erg} \mathrm{s}^{-1}$ (as indicated by the wavy line).

area in Fig. 4, where it is compared with the luminosity function of local sources taken from Sazonov et al. (2006).

As follows from Fig. 4, the allowed range of luminosity functions in the GP region is fully compatible with the luminosity function of point sources in the Solar vicinity. It is important to note here that the $\sim 100 \mathrm{ks}$ Chandra observation of the GP region allows us to efficiently constrain the luminosity function only in the range $10^{30}-10^{32} \mathrm{erg} \mathrm{s}^{-1}$. The upper bound appears due to the limited stellar mass within the small solid angle covered by Chandra $\left(<\right.$ few $\left.\times 10^{6} M_{\odot}\right)$. Sources brighter than $\sim 10^{32} \mathrm{erg} \mathrm{s}^{-1}$ are simply too rare to be found in such a small area of the Galactic disk. The lower bound is due to finite Chandra's sensitivity.

In Fig. 5 we compare the cumulative $\log N-\log S$ distributions constructed for the GP field and for the CDFS and CDFN (all corrected for the incompleteness at low count rates). One can clearly see how Galactic sources become progressively more dominant toward lower fluxes.

\subsection{Resolved fraction of the GRXE}

Fraction of the energy flux resolved into point sources in a relatively broad energy range that we consider (1-7 keV) depends on the spectral shapes both of the Galactic ridge and the cumulative emission of point sources. Due to very strong and variable (for example due to different distances to sources) interstellar absorption of the X-rays in the region of our study it is very hard to predict the cumulative spectrum of point sources independently. Therefore in the following estimations of the fraction of the emission resolved into point sources we can refer either simply to Chandra count rates in the energy band $1-7 \mathrm{keV}$, or to count rate converted into energy fluxes using some fiducial shape of sources cumulative spectrum.

Integrating the number-flux function measured in the GP region (Fig. 5) down to our effective detection limit of 5 counts (over the $102 \mathrm{ks}$ exposure, which corresponds to an absorption corrected flux of $1.2 \times 10^{-15} \mathrm{erg} \mathrm{s}^{-1} \mathrm{~cm}^{-2}$ in the $1-7 \mathrm{keV}$ band for Galactic sources, assuming a power low spectral shape

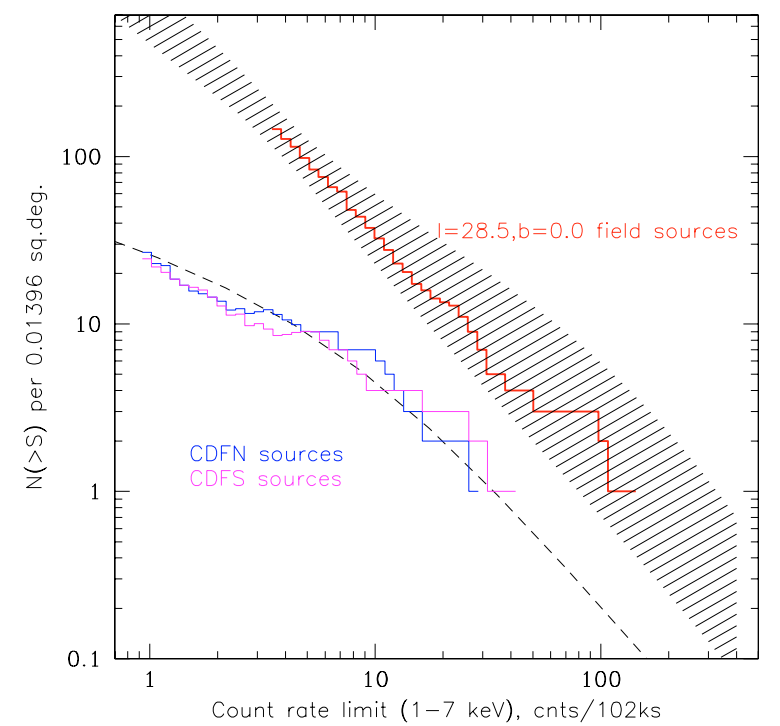

Fig. 5. Cumulative number-flux functions of point sources detected in the GP region (upper histogram) and in extragalactic fields (Chandra Deep Fields North and South). All data (GP, CDFN and CDFS) are corrected for the incompleteness at low count rates. The dashed line shows an approximation of the extragalactic source counts by a simple analytic model similar to that of Moretti et al. (2002).

with $\Gamma=2.0$ and $N_{\mathrm{H}}=2 \times 10^{22} \mathrm{~cm}^{-2}$ ) yields $\sim 1.6 \mathrm{kcnts}$ out of the total $\sim 6.5 \mathrm{kcnts}$ collected by Chandra. This implies that $\sim 25 \%$ of the total flux at energies $1-7 \mathrm{keV}$ from the GP region is already resolved by Chandra into point sources. If we similarly integrate the expected $\log N-\log S$ function of extragalactic sources (aslo shown in Fig. 5) down to the same count rate limit (which corresponds to a somewhat higher absorption corrected flux of $2.5 \times 10^{-15} \mathrm{erg} \mathrm{s}^{-1} \mathrm{~cm}^{-2}$ for $N_{\mathrm{H}} \sim 6 \times 10^{22} \mathrm{~cm}^{-2}$ ), we find that $\sim 6 \%$ of the total flux is resolved into extragalactic sources.

This implies that at least $\sim 19 \%$ of the GRXE is due to Galactic point sources, presumably cataclysmic variables and coronally active stars. Moreover, an extrapolation of the measured $\log N-\log S$ curve to fluxes below the current detection limit based on the luminosity function of local weak X-ray sources (Sazonov et al. 2006) is consistent with all of the X-ray flux from the GP being due to point sources, mostly of Galactic origin. These conclusions are consistent with our results for the Galactic Center region (Revnivtsev et al. 2006c).

We should say in conclusion a few words about the apparent difference of our results from those of Ebisawa et al. (2005). We can mention two main differences in the approaches taken. First, Ebisawa et al. (2005) assumed some fiducial luminosity functions of weak Galactic X-ray sources and some fiducial mass model of the Galaxy while predicting the number-flux function of possible galactic sources. In our paper we use the luminosity function of weak sources measured in the Solar neighborhood (Sazonov et al. 2006), which is quite different from the fiducial functions of Ebisawa et al. (2005), and demonstrate that this luminosity function is fully consistent with the number-flux function of sources masured in the GP region. Also the mass model of the Galaxy was specially checked to provide correct NIR flux in the direction of the study ensuring the more or less correct overlooked mass of stars. Second, in order to increase the sensitivity to weak point sources, we used an optimized broad energy band, which includes softer energies (1-2 keV), where Chandra has the maximum sensitivity to the galactic point sources, used only the central region of the Chandra field additionally maximizing 
our sensitivity to point sources and also we took into account the effects of the Possion noise on the observed number-flux function. All together these efforts allowed us to move deeper in sensitivity and to make more accurate comparison with the point sources hypothesys of the formation of the ridge emission.

Acknowledgements. This research made use of data obtained from the High Energy Astrophysics Science Archive Research Center Online Service, provided by the NASA/Goddard Space Flight Center. We acknowledge the use of the Legacy Archive for Microwave Background Data Analysis (LAMBDA). Support for LAMBDA is provided by the NASA Office of Space Science. MR aknowledge partial support of grant RFFI 07-02-00961.

\section{References}

Bauer, F. E., Alexander, D. M., Brandt, W. N., et al. 2004, AJ, 128, 2048 Bleach, R. D., Boldt, E. A., Holt, S. S., Schwartz, D. A., \& Serlemitsos, P. J. 1972, ApJ, 174, L101

Cappelluti, N., et al. 2007 [arXiv: astro-ph/0701196]

Cooke, B. A., Griffiths, R. E., \& Pounds, K. A. 1970, IAUS, 37, 280

Dickey, J. M., \& Lockman, F. J. 1990, ARA\&A, 28, 215

Ebisawa, K., et al. 2005, ApJ, 635, 214

Eisenhauer, F., et al. 2005, ApJ, 628, 246

Freudenreich, H. T. 1998, ApJ, 492, 495

Hasinger, G., Burg, R., Giacconi, R., et al. 1993, A\&A, 275, 1

Hickox, R. C., \& Markevitch, M. 2006, ApJ, 645, 95

Kenter, A. T., \& Murray, S. S. 2003, ApJ, 584, 1016

Koyama, K., Makishima, K., Tanaka, Y., \& Tsunemi, H. 1986, PASJ, 38, 121

Koyama, K., Awaki, H., Kunieda, H., Takano, S., \& Tawara, Y. 1989, Nature, 339,603
Koyama, K., et al. 2006 [arXiv: astro-ph/0609215]

Krivonos, R., Revnivtsev, M., Churazov, E., et al. 2006

[arXiv: astro-ph/0605420]

Launhardt, R., Zylka, R., \& Mezger, P. G. 2002, A\&A, 384, 112

Moretti, A., Lazzati, D., Campana, S., \& Tagliaferri, G. 2002, ApJ, 570, 502

Mukai, K., \& Shiokawa, K. 1993, ApJ, 418, 863

Muno, M. P., Bauer, F. E., Bandyopadhyay, R. M., \& Wang, Q. D. 2006, ApJS, 165,173

Ottmann, R., \& Schmitt, J. H. M. M. 1992, A\&A, 256, 421

Revnivtsev, M., Gilfanov, M., Sunyaev, R., Jahoda, K., \& Markwardt, C. 2003, A\&A, 411, 329

Revnivtsev, M., Gilfanov, M., Jahoda, K., \& Sunyaev, R. 2005, A\&A, 444, 381 Revnivtsev, M., Molkov, S., \& Sazonov, S. 2006a, MNRAS, 373, L11

Revnivtsev, M., Sazonov, S., Gilfanov, M., Churazov, E., \& Sunyaev, R. 2006b, A\&A, 452, 169

Revnivtsev, M., Vikhlinin, A., \& Sazonov, S. 2006c

[arXiv:astro-ph/0611952]

Robin, A. C., Reylé, C., Derrière, \& Picaud, S. 2003, A\&A, 409, 523

Sazonov, S., Revnivtsev, M., Gilfanov, M., Churazov, E., \& Sunyaev, R. 2006, A\&A, 450, 117

Schmitt, J. H. M. M., Collura, A., Sciortino, S., et al. 1990, ApJ, 365, 704

Tanaka, Y. 2002, A\&A, 382, 1052

Vaiana, G. S. 1981, SSRv, 30, 151

Vikhlinin, A., McNamara, B. R., Forman, W., et al. 1998, ApJ, 502, 558

Vikhlinin, A., Markevitch, M., Murray, S. S., et al. 2005, ApJ, 628, 655

Voss, R., \& Gilfanov, M. 2006, A\&A, 447, 71

Worrall, D. M., Marshall, F. E., Boldt, E. A., \& Swank, J. H. 1982, ApJ, 255, 111

Worrall, D. M., \& Marshall, F. E. 1983, ApJ, 267, 691

Warwick, R. S., Turner, M. J. L., Watson, M. G., \& Willingale, R. 1985, Nature, 317, 218

Yamauchi, S., \& Koyama, K. 1993, ApJ, 404, 620 